\title{
Das Sekundärressourcenpotenzial aus Windkraft- und Photovoltaikanlagen
}

\author{
Silvia Scherhaufer (D) - Florian Part · Peter Beigl
}

Angenommen: 15. Oktober 2020 / Online publiziert: 10. November 2020 (C) Der/die Autor(en) 2020

\begin{abstract}
Zusammenfassung Bis 2040 soll die Stromversorgung in Österreich laut aktuellem Regierungsprogramm klimaneutral sein und somit $\mathrm{zu} 100 \%$ aus erneuerbarer Energie erfolgen. Dieses Ziel ist mit großen Ambitionen im Ausbau der Anlagen zur Erzeugung von erneuerbarer Energie, vor allem im Bereich der Photovoltaik und Windkraft, verbunden. Ziel dieses Beitrags

winnungsraten deutlich gesteigert werden können. Aufgrund der anfallenden Mengen und Qualitäten an potenziellen Sekundärressourcen, die in den nächsten Jahrzehnten in den abgebauten Anlagen enthalten sein werden, müssen optimierte Verwertungswege geschaffen werden, damit die Ressourcen auch in der Praxis im Kreislauf geführt werden können.
\end{abstract} ist es, den zu erwartenden, stark ansteigenden Materialeinsatz für neue und bestehende Windkraftanlagen (WKA) und Photovoltaikanlagen (PVA) bis zum Jahr 2050 darzustellen, das Potenzial an Sekundärressourcen abzuschätzen und Wege zur Wiederverwendung und möglichst hochwertigen Verwertung aufzuzeigen. Hierfür wurden der Anlagenbestand und die verwertbaren Sekundärressourcen auf Basis von makroökonomischer Modellierung des österreichischen Energiesystems und marktmixbasierten Materialflussanalysen von WKA und PVA (auf Bauteilebene) bilanziert. Beim Bau der Anlagen zeigt sich die Massenrelevanz von Silizium und Glas bei PVA bzw. Beton und Stahl für Fundamente für WKA, wobei im Zeitraum von 2020 bis 2030 mit einer Steigerung des Rohstoffeinsatzes um den Faktor 5 zu rechnen ist. Weiters enthalten elektrische und elektronische Bauteile bedeutende Mengen an Metallen ( $\mathrm{Fe}, \mathrm{Cu}, \mathrm{Al}$ ), Edelmetallen $\mathrm{Au}, \mathrm{Ag})$ sowie Sondermetallen, wie Tantal in Kondensatoren oder Neodym in Magneten. Ein Vergleich nach zwei Recyclingszenarien mit derzeitiger Ausrichtung und hochwertigem Recycling zeigt, dass bei PVA für Glas, Silizium und Silber bzw. bei WKA bei Kupfer, Edelmetallen und glas- und carbonfaserverstärkten Kunststoffen Rückge-

DI (FH) S. Scherhaufer $(\triangle)$

DI Dr. F. Part · DI Mag. P. Beigl Institut für Abfallwirtschaft,

Universität für Bodenkultur Wien,

Muthgasse 107/III, 1190 Wien,

Österreich

silvia.scherhaufer@boku.ac.at
Schlüsselwörter Massenflussanalyse · Sekundärressourcen · Erneuerbare Energie $\cdot$ Energiewende

\section{The potential of secondary resources from wind power and photovoltaic plants}

Abstract According to the current government program, the electricity supply in Austria is to be climate-neutral by 2040 and thus be $100 \%$ renewable energy. This goal is connected with great ambitions in the expansion of renewable energy production facilities, especially in the area of photovoltaics and wind power. The aim of this paper is to present the expected sharp increase in the use of materials for new and existing wind power plants (WPP) and photovoltaic plants (PVP) by 2050, to estimate the potential of secondary resources and to show ways for reuse and the highest possible level of recycling. The plant stock and the usable secondary resources were balanced on the basis of macroeconomic modelling of the Austrian energy system and market mix based material flow analyses of WPP and PVP on component level. The construction of the plants shows the mass relevance of silicon and glass for PVP and concrete and steel for foundations of WPP, whereby an increase in the use of raw materials by a factor of 5 can be expected in the period from 2020 to 2030 . Furthermore, electrical and electronic components contain significant amounts of metals $(\mathrm{Fe}, \mathrm{Cu}, \mathrm{Al})$, precious metals $(\mathrm{Au}$, $\mathrm{Ag}$ ) and special metals such as tanta- lum and neodymium in capacitors and magnets. A comparison after two recycling scenarios with current state-ofthe-art and high quality recycling shows that recovery rates can be significantly increased for PVP concerning glass, silicon and silver and for WPP concerning copper, precious metals and glass and carbon fibre reinforced plastics. Due to the quantities and qualities of potential secondary resources from dismantled plants in the coming decades, optimized recycling paths must be created so that the resources can be managed in a circular economy.

Keywords Mass Flow Analysis . Secondary resources $\cdot$ Renewable energy $\cdot$ Energy transition

\section{Einleitung}

Die steigende Nachfrage nach Ressourcen zeigt zunehmend negative Auswirkungen auf die Umwelt und gefährdet gleichzeitig das Wohlergehen der Bevölkerung. Der Abbau und die Verarbeitung von Rohstoffen und die Weiterverarbeitung zu Produkten erfordern einen hohen Rohstoff- und Energieeinsatz und belasten damit das Klima. Zudem korreliert die Gewinnung und Aufbereitung abiotischer Rohstoffe in den Bergbauländern oft mit der Nutzung von Chemikalien und Wasser, wodurch Trinkwasserressourcen, Boden und Luft kontaminiert und die Lebensgrundlagen der lokalen Bevölkerung gefährdet werden. Angesichts der begrenzten Verfügbarkeit natürlicher Ressourcen und der mit ihrer Nutzung verbundenen Umweltauswirkungen wird deutlich, dass das Muster der Ressourcennutzung in den Industrieländern weder nachhaltig noch weltweit übertragbar ist. Daher besteht dringender Handlungsbedarf, um den derzeitigen Primärrohstoffverbrauch zu minimieren bzw. Sekundärrohstoffe im Sinne der Circular Economy (CE) rückzugewinnen. So werden beispielsweise in Windkraftanalagen und Energie- 
zwischenspeichern kritische Rohstoffe (z.B. Neodym, Lithium etc.) in großen Mengen verbaut, die ökonomisch sehr wichtig für die europäische Wirtschaft sind. Eine CE-Strategie soll somit eine nachhaltige, $\mathrm{CO}_{2}$-arme, ressourceneffiziente und wettbewerbsfähige Wirtschaft in der EU schaffen (Europäische Kommission 2015). Verfahren zur Rückgewinnung von Sekundärrohstoffen spielen hierbei eine wesentliche Rolle, da sie unmittelbar auf die Quantität und Qualität von Recyclingprodukten wirken. So wurde im Sinne des „EU Green Deals“ und der CE ein Aktionsplan (European Commission 2020a) ins Leben gerufen, um europäische Produkte sauberer und kompetitiver $\mathrm{zu}$ gestalten.

Demnach soll eine Dekarbonisierung stattfinden, um eine globale Erwärmung einzudämmen bzw. die Pariser Klimaziele zu erreichen. Die Strategie zur Energiewende beinhaltet neben Maßnahmen zur Energieeinsparung auch die Steigerung der Stromproduktion aus erneuerbaren Energiequellen. Im aktuellen Regierungsprogramm soll Österreich bis 2040 klimaneutral werden (Österreichische Bundesregierung 2020). Größtes Ausbaupotenzial bezüglich der Stromproduktion aus erneuerbarer Energie besteht in Österreich bei Windkraftanlagen (WKA) und Photovoltaikanlagen (PVA), während der Ausbau im Bereich der Wasserkraft an Kapazitätsgrenzen stößt. Nach aktuellen Schätzungen (Biermayr et al. 2020; Photovoltaic Austria 2020) sollen bis 2030 die Stromerzeugung aus PVA von derzeit rund 1,7 Terawattstunden (TWh) auf $13 \mathrm{TWh}$ und aus Windkraft von derzeit rund 7 auf 22,5 TWh erhöht werden. Anteile der PV- bzw. Wind-Energie an der Stromaufbringung würden 2030 in etwa $15 \%$ bzw. $26 \%$ betragen. Neben Wasserkraft wären sie somit die wichtigsten erneuerbaren Energiequellen in Österreich.

Mit Hinblick auf die CE-Strategie wird in diesem Fachbeitrag der Materi- aleinsatz in WKA und PVA näher analysiert. Dieser Beitrag soll einen Überblick über den Rohstoffbestand in bereits installierten WKA und PVA geben und zukünftige Entwicklungen mit stark steigenden jährlichen Ausbauraten hinsichtlich der ambitionierten Klimaziele mitberücksichtigen. Es soll als Grundlage dienen, um zukünftige quantitative und qualitative Abschätzungen der Sekundärressourcenpotenziale aus WKA und PVA tätigen zu können. Übergeordnetes Ziel wäre es, die Wege des Abfallbewirtschaftungssystems so $\mathrm{zu}$ gestalten, dass möglichst hochwertige Sekundärrohstoffe produziert werden können, um zum einen eine hohe Versorgungssicherheit in Europa zu gewährleisten und zum anderen Rohstoffe so lange und werterhaltend wie möglich im Kreislauf zu halten.

\section{Material und Methoden}

\subsection{Vorgehensweise}

Ziel der Studie ist, das Sekundärressourcenpotenzial zur Rückgewinnung aus dem derzeitigen Bestand an PKA und WKA zu ermitteln. Zur Ermittlung der eingesetzten Ressourcen innerhalb der Systeme wird eine Materialflussanalyse (MFA) durchgeführt (Abb. 1). Laut ÖNORM S 2096/2005 befasst sich die MFA mit der „Identifizierung und Quantifizierung aller relevanten Flüsse von Materialien in einem zeitlich und räumlich exakt abgegrenzten System sowie Bilanzierung der Materialien innerhalb dieses Systems“. Die Identifizierung aller relevanten Materialflüsse im betrachteten System erfolgt mittels „Bottom-up“-Ansatzes, um den derzeitigen Bestand an verbauten Rohstoffen sowie in weiterer Folge den zukünftigen Bestand hochrechnen zu können.

Die Materialzusammensetzung zwischen den eingesetzten Produkttypen kann stark variieren, weshalb es erforderlich war, die verwendeten Produkttypen in Österreich anhand der

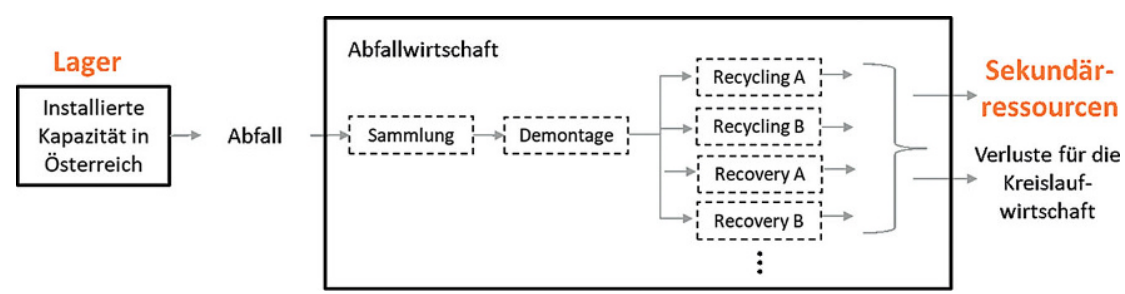

Abb. 1 Schematische Darstellung der Materialflussanalyse mit der Systemgrenze installierte PVA und WKA in Österreich von 2020 bis 2040
Marktmengen zu bestimmen. Dazu wurde die Marktsituation in Österreich näher untersucht und ein Marktmix aus den eingesetzten Technologien bei PVA (Dünnschicht- oder siliziumbasierte Module) und WKA (mit Getriebe oder getriebelos) erstellt, welcher durch bestimmte Indikatorprodukte (z.B. ein 1,6 $\mathrm{m}^{2}$ PV-Modul mit 60 Siliziumzellen zu je $242 \mathrm{~cm}^{2}$ und einer Dicke von $200 \mu \mathrm{m})$ abgebildet wurde. Indikatorprodukte wurden herangezogen, um den Datenpool der Materialzusammensetzung der einzelnen Produkte einzuschränken. Materialintensität und -zusammensetzung wurden für die identifizierten Indikatorprodukte mit Schwerpunkt auf Metalle, Kunststoffe und sonstige massenrelevante Fraktionen ermittelt. Die Materialzusammensetzung wurde in einem stufenweisen Verfahren bestimmt, beginnend mit der Zusammensetzung der Hauptkomponenten über die spezifischen Subkomponenten bis hin zu einem detaillierten Materialkatalog einzelner Bestandteile. Somit erfolgt die MFA sowohl auf der sogenannten Güter- als auch auf Stoffebene.

Die identifizierten Indikatorprodukte sollen die Marktsituation in Österreich abbilden. Die Auswahl für den Marktmix basiert auf installierten Anlagen im Jahr 2019, wobei die Trends der Vorjahre ab 2010 berücksichtigt werden. Um Informationslücken zu schließen, wurden Interviews mit ExpertInnen der PV-Branche in Österreich (z. B. PV Austria, FH Technikum Wien) sowie der Wind-Branche - vor allem WindAnlagen-Betreiber - durchgeführt, insbesondere zu Themen wie der Größe der installierten PV-Anlagen, zur Art der installierten Montagesysteme, zur Situation der PV-Heimspeicheranlagen, zum Einsatz von getriebelosen WKA oder WKA mit Getriebe sowie zur zukünftigen Entwicklung.

Die Materialzusammensetzung in Kombination mit dem in Österreich verwendeten Marktmix und den installierten Anlagen führen zu Materialbestandsströmen, die derzeit in Österreich installiert sind. Dieser WKAund PVA-Bestand wird in der vorliegenden Arbeit als (anthropogenes) „Lager" bezeichnet. Da das Gesamtziel des Projekts darin besteht, das Sekundärressourcenpotenzial innerhalb der Landesgrenzen $\mathrm{zu}$ ermitteln, werden alle Massenströme auf die installierte Leistung bezogen. Die Bezugsgröße für PV wurde folglich als Watt peak (Wp) 


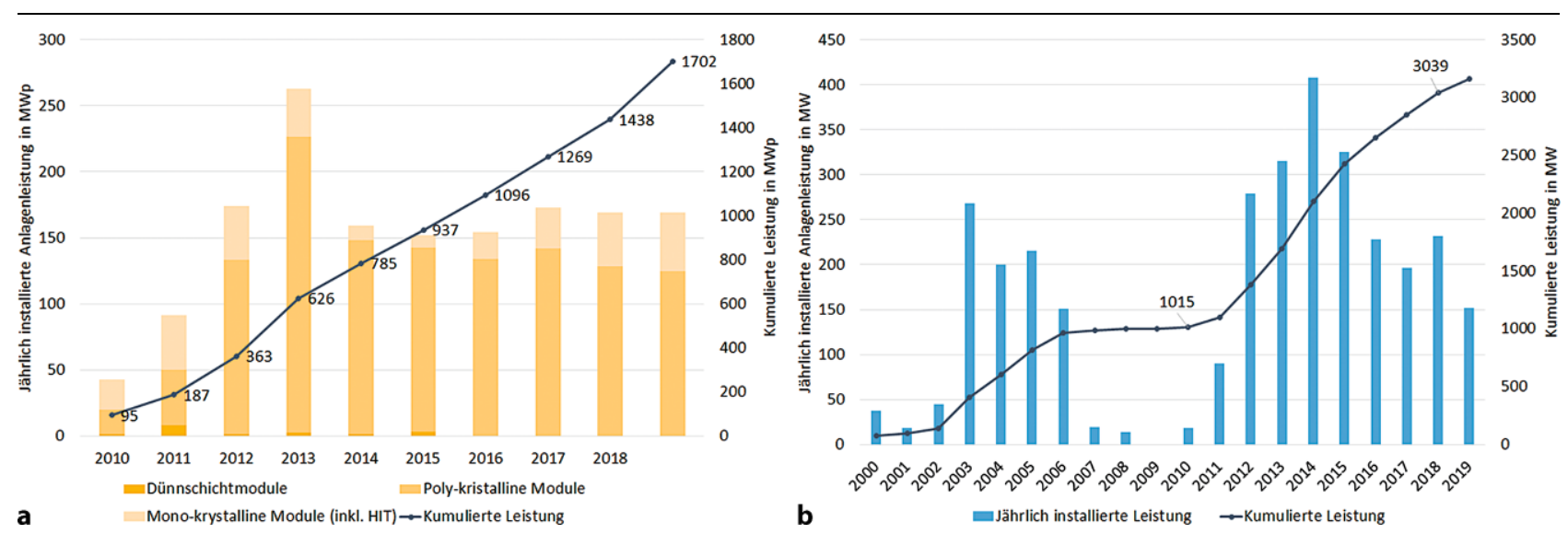

Abb. 2 Entwicklung von Bestand und Neuinstallationen von PVA (a) und WKA (b) in Österreich nach Biermayr et al. (2020)

herangezogen, welche die maximale (Spitzen-)Leistung von PV-Modulen unter Testbedingungen abbildet. Für Windenergie wurde die Bezugsgröße Watt (W) herangezogen. Das Potenzial an Sekundärressourcen, die bei Abfallende bzw. End-of-Life (EoL) der Anlage theoretisch für die Rückgewinnung zur Verfügung stehen würde, wird anhand von mehreren Recyclingszenarien bestimmt. Dabei wird auf das spezifische Sammelsystem nicht näher eingegangen und so wird die Rücklaufquote vereinfacht mit $100 \%$ angesetzt.

Fokus für die Identifizierung von recyclingfähigen Materialien bzw. Stoffgruppen liegt auf Metallen wie Stahl, Aluminium und Kupfer, Edel- und Sondermetallen wie Gold, Silber, Palladium und Tantal, kritischen Rohstoffen wie Lithium und Kobalt sowie massenreichen Fraktionen wie glasfaser- oder carbonfaserverstärkter Kunststoff (GFK bzw. CFK). Dabei wird das Ziel des Kreislaufwirtschaftspakets berücksichtigt, diese Sekundärrohstoffe wie Primärrohstoffe als anthropogenes Lager $\mathrm{zu}$ handeln. Das stoffliche Recycling ist dabei im Vordergrund. Alle anderen möglichen Entsorgungswege, wie das thermische Recycling, werden als Verluste für die Rohstoff-Wirtschaft angeführt.

\subsection{Untersuchungsgegenstand und Systemgrenzen}

Für die zwei betrachteten Produktsysteme - PVA und WKA - werden soweit möglich alle Komponenten bzw. Bestandteile berücksichtigt, die für den Betrieb dieser Anlagen notwendig sind, die gleichzeitig auch die Systemgrenzen der MFA bilden. Der Untersuchungsrahmen einer PVA schließt daher das
PV-Modul, aber auch die Vorrichtungen für die Montage der Module, die eingesetzten elektronischen und elektrischen Bauteile (Wechselrichter, Laderegler), Kabel und gegebenenfalls einer Batterie mit ein. Bei WKA werden sowohl der Turm, die Gondel und Rotorblätter, als auch die eingebaute Elektronik, der Transformator sowie das für die Statik notwendige Fundament mitberücksichtigt.

Die Anzahl der jährlich installierten PV-Module erreichte 2013 mit über 250 MWp einen Höchststand und blieb dann mit jährlichen Installationsraten zwischen 150 und 160 MWp konstant (Abb. 2a). Bis Ende 2019 wurde eine kumulierte Anzahl von installierten PVAnlagen in Österreich von insgesamt 1,7 GWp erreicht. Installierte Paneltypen in Österreich sind vor allem polykristalline Siliziumzellen mit einem Marktanteil von $74 \%$, gefolgt von monokristallinen Siliziumzellen mit $26 \%$ (Biermayr et al. 2020). Sogenannte HIT-Solarzellen („Heterojunction with Intrinsic Thin-Layer") werden unter der Kategorie monokristalliner Zellen angeführt, da ihr interner Zellaufbau neben kristallinem auch amorphes Silizium beinhaltet, sie aber über andere Elektrodenmaterialen verfügen. Dünnschichtzellen aus Cadmium-Tellurid (CdTe) oder Kupfer-Indium-GalliumDiselenid (CIGS) haben in Österreich mit einem Marktanteil von unter $1 \%$ eher eine geringe Bedeutung. Darüber hinaus spielt der Einsatz von Energiespeichersystemen zur Erhöhung des Eigenstromverbrauchs eine immer größere Rolle. 2017 wurden bereits $30 \%$ der neu installierten PV-Anlagen mit Energiespeichersystemen versehen (Fischer and Leohnhartsberger 2019).
WKA hatten in den Jahren 2003 bis 2006 einen ersten Boom in Österreich und erreichten nach einem Ausfall aufgrund der Wirtschaftskrise 2014 einen Höchststand bei Neuinstallationen (Abb. 2b). Die Windenergie war in den letzten 10 Jahren eine wachsende Branche in Österreich und erreichte 2019 eine installierte Nennleistung von mehr als $3 \mathrm{GW}$, die in insgesamt 1340 Anlagen produziert werden. Ein Trend zu einer geringeren spezifischen Generatorleistung und größeren Rotordurchmessern wurde beobachtet. Lag die durchschnittliche Anlagenleistung der neu installierten Anlagen 2004 noch bei $1,78 \mathrm{MW}$ pro Turbine, waren es 2013 bereits 2,73 MW pro Turbine (Winkelmeier et al. 2014). Die in den letzten Jahren hauptsächlich installierten WKA betrugen in Österreich $3 \mathrm{MW}$ pro Anlage (Biermayr et al. 2020). Es ist auch zu erwähnen, dass in Österreich das sogenannte Repowering (der Ersatz von alten durch neue WKA in bestehenden Windkraftparks) an Bedeutung zunimmt. So werden beispielsweise im Burgenland 26 Windräder mit einer Gesamtleistung von 46,8 MW, die 2003 errichtet wurden, durch 13 neue WKA ausgetauscht und die Gesamtleistung gleichzeitig auf $65 \mathrm{MW}$ erhöht. Zusammengefasst sind zwei WKA-Hauptgruppen in Österreich installiert: Anlagen mit Getriebe und getriebelose Anlagen mit Direktantrieb. Aktuell sind ca. zwei Drittel der installierten WKA ohne Getriebe und ein Drittel mit Getriebe (Biermayr et al. 2020). Nach IG Windkraft dokumentiert in Einsiedler (2017) sind in Österreich mit Stand 2017 nur 9 Windkraftanlagen mit Permanentmagneten, die den kritischen Rohstoff Neodym (Nd) beinhalten, in Betrieb. 


\subsection{Materialflüsse und Datengrundlage}

Die Daten zur Materialzusammensetzung wurden hauptsächlich aus Ökobilanzstudien (Life Cycle Assessment) entnommen, da sie eine gute Dokumentation der Daten darstellen, die in den jeweiligen Ökobilanz-Inventaren (Life Cycle Inventories) verwendet werden. Der Unterschied zwischen den für eine Ökobilanz verwendeten Daten und den in dieser Studie verwendeten Massenströmen besteht darin, dass dieses Ökobilanz-Inventar neben dem Materialeinsatz auch Reinigungs- oder Betriebsmittel, Lötflussmittel, Verpackungsmaterialien und den Energiebedarf für die Herstellung, Materialverluste und Abfälle enthalten. Für diese MFA sind somit nur Materialien erfasst, die in den Produktsystemen eingebaut sind.

Die Materialzusammensetzung kann je nach PVA-Typ aufgrund unterschiedlicher Konstruktionen und unterschiedlicher Verwendung von Art und Menge der Materialien bzw. von Windturbinen-Größe und -Art variieren. Folglich können die Datenbestände in einzelnen Studien sehr stark voneinander abweichen. Daten zu multikristallinen Siliziummodulen liegen vorwiegend in Kranert et al. (2012), Jungbluth et al. (2009), De Wild-Scholten (2014) und Frischknecht et al. (2015) vor. Die Quelle der in Kranert et al. (2012) berichteten Daten ist jedoch unbekannt. Dagegen werden die Daten von Jungbluth et al. (2009) in De Wild-Scholten (2014) hinsichtlich der reduzierten Dicke der Wafer (Halbleiterscheiben), verbesserter Umwandlungseffizienz und neuartiger Silizium-Rohstoffe und Waferprozesse aktualisiert. Frischknecht et al. (2015), ein Bericht des Photovoltaic Power Systems Programme (IEA PVPS) der Internationalen Energieagentur, bezieht sich wiederum auf Daten von De Wild-Scholten (2014). Als Schlussfolgerung wurden für diese Studie Daten von Frischknecht et al. (2015) verwendet.

Ökobilanzen zu HIT-Zellen sind bei Louwen et al. (2015) und Olson et al. (2011) verfügbar. Für die HIT wurde ein monokristalliner Silizium-Wafer verwendet. In beiden Publikationen wurden Ökoinventar-Daten (Ecoinvent) verwendet und die Materialzusammensetzung von HIT ähnelt den in Stolz et al. (2017) berichteten monokristallinen Zellen. Der Zellwirkungsgrad wurde jedoch auf $20 \%$ angepasst, wie in Louwen et al. (2015) berichtet.
Die Materialzusammensetzung eines Wechselrichters mit 2500W Nennleistung bei einem durchschnittlichen Gesamtgewicht von $18,5 \mathrm{~kg}$ wurde aus der in Jungbluth et al. (2009) angegebenen Zusammensetzung verwendet. Ergänzt wurde die Zusammensetzung durch die Material- und Massenanteile der elektronischen Komponenten (Kondensatoren, Leiterplatten etc.) aus Hischier et al. (2007a; 2007b). Auf dieser Basis konnte die Kennzahl von $10 \mathrm{~kg}$ Wechselrichter pro kWp ermittelt werden.

Ökobilanzstudien zu WKA gibt es beispielsweise von Ardente et al. (2008) über eine italienische Windfarm mit 11 Turbinen von je $660 \mathrm{~kW}$, von Guezuraga et al. (2012) über eine 2-MWTurbine mit Getriebe und einer getriebelosen 1,8-MW-Turbine, aber auch von den Herstellern selbst wie von Garrett and Rønde (2014) und Vestas (2015) über 2-MW-Turbinen sowie von Vestas (2017) über 3,45-MW-Turbinen. Alle Studien haben gemeinsam, dass die Zusammensetzung nur auf Ebene der Haupt- und nicht der Subkomponenten angegeben wird. Um die österreichische Situation bestmöglich abzudecken, wurden die aktuellsten Daten aus Vestas (2017) herangezogen, da der Hersteller Vestas nach Enercon die größte Marktrelevanz in Österreich hat. Weiters trägt diese Studie dem Trend zu höherer MW-Leistung pro Turbine Rechnung. Die Nabenhöhe der VestasTurbinen liegt bei $94 \mathrm{~m}$ und der Rotordurchmesser bei $112 \mathrm{~m}$. Diese Größen sind für die installierten Anlagen in Österreich repräsentativ, da mittlerweile der durchschnittliche Rotordurchmesser der neuen installierten Anlagen bei $114 \mathrm{~m}$ und die Turmhöhe bei etwa $120 \mathrm{~m}$ liegt. Da die Elektronik zwar in der Studie von Vestas (2017) berücksichtigt wurde, allerdings nicht in der gewünschten Detailtiefe der Subkomponenten aufscheint, müssen die elektronischen Systeme mit Daten aus Hischier et al. (2007a; 2007b) komplettiert werden. Außerdem muss für den österreichischen Mix auch noch der Turm angepasst werden, da auch immer mehr Hybrid-Türme (Stahl und Beton) installiert werden. Nach Angaben von Interviewpartnern in dieser Branche werden bei einer Höhe von $>120 \mathrm{~m}$ tendenziell eher Hybrid-Türme (Stahl und Beton) bzw. bei $<120 \mathrm{~m}$ Stahltürme errichtet.

\subsection{Szenarien}

Für die vorliegende Arbeit wurden zwei mögliche Szenarien zur Bestandsentwicklung bis zum Jahr 2040 herangezogen sowie zwei mögliche Szenarien zum Recycling.

\subsubsection{Szenarien zur}

\section{Bestandsentwicklung}

Ausgehend vom Jahr 2019 wird die Bestandsentwicklung bis 2040 unter Zuhilfenahme der vom Umweltbundesamt (Umweltbundesamt 2018, 2019) entwickelten Szenarien „WEM“ („,with existing measures") und „Transition“ abgeschätzt. Im WEM-Szenario werden alle bis zum Stichtag 1. Jänner 2018 verbindlich umgesetzten Maßnahmen berücksichtigt. Das Transition-Szenario umfasst Klimaschutzmaßnahmen, welche die mittel- bis langfristigen Ziele der europäischen Klimapolitik umsetzen (d.h. Reduktion der energiebedingten THG-Emissionen in Österreich um mindestens 80 bis $95 \%$ bis 2050) und welche von den wichtigsten Klimaund Energie-Akteuren aus Österreich akzeptiert werden. Das WEM- und Transition-Szenario werden in einem modellgestützten, multidisziplinären Bewertungs- und Quantifizierungsmodell, welches in Meyer et al. $(2018,2020)$ näher beschrieben wird, hinsichtlich der Auswirkungen auf das Bruttoinlandsprodukt und die Beschäftigung ökonomisch analysiert. Dafür wurde zum Zweck der Modellierung des österreichischen Energiesystems das makroökonomische Top-Down-Modell WIFO.DYNK („Dynamischer Neu-Keynesianer") (Kirchner et al. 2017; Kratena and Sommer 2014) mit dem Bottomup-Modell der Österreichischen Energieagentur (TIMES) für Szenarien der Elektrizitäts- und Wärmeerzeugung aus Kraftwerken kombiniert.

Gemäß aktuellem Regierungsprogramm soll Österreich bis 2040 klimaneutral sein (Österreichische Bundesregierung 2020). Deshalb wurden die WEM- und Transition-Szenarien, die ursprünglich bis 2050 bemessen waren, auf 2040 komprimiert. Außerdem beruhen die Startwerte im Jahr 2020 auf Echtwerten vom Jahr 2019 und der Annahme, dass der Ausbau im Mittel der letzten 5 Jahre liegt (Abb. 3). 
WEM 2040

25,000

20,000

琣 15,000

5,000
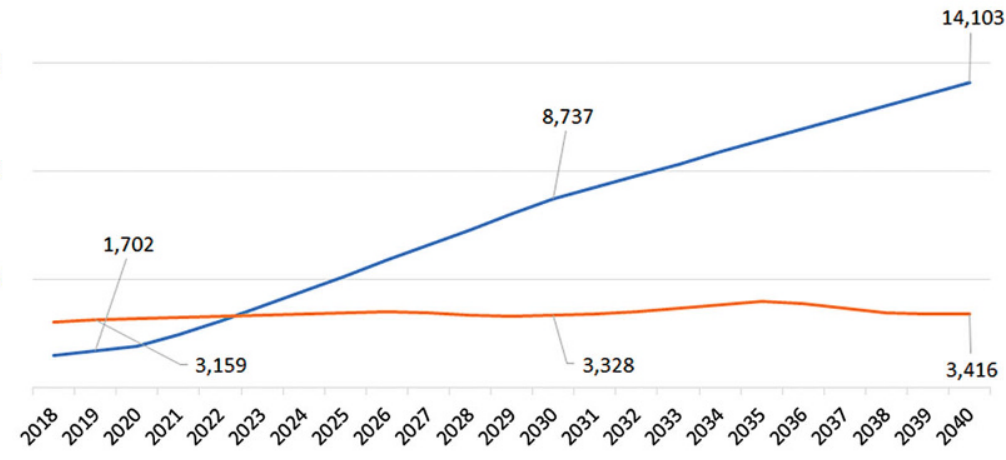

a

-Photovoltaik —Wind

Transition 2040

25,000

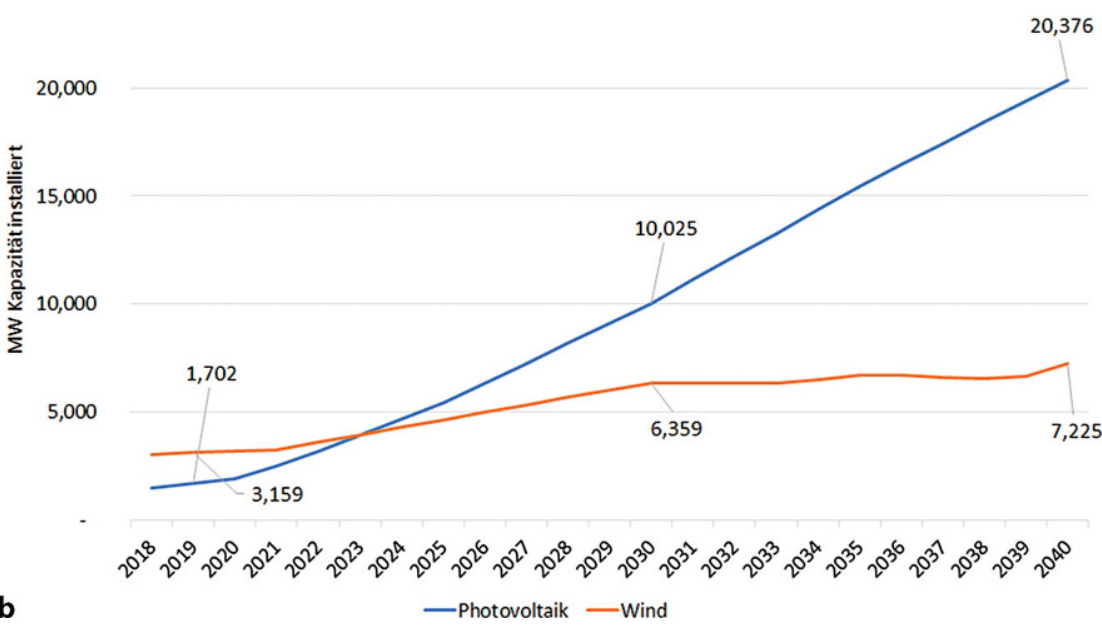

Abb. 3 Entwicklung derinstallierten Leistung vonPVA undWKAinden SzenarienWEM und Transition komprimiert auf Ziel Klimaneutralität 2040, basierend auf den makroökonomischen Modellen WIFO.DYNK und TIMES

\subsubsection{Recyclingszenarien}

Derzeit befinden sich die meisten WKA und PVA noch in Betrieb und mussten bisher noch nicht abgerissen bzw. entsorgt werden. Um die zukünftige Abfallmenge prognostizieren zu können, kann im Allgemeinen von einer funktionellen Lebensdauer für PVA von 30 Jahren und für WKA von 20 bis 30 Jahren ausgegangen werden (Bio Intelligence Service 2011; Stolz et al. 2017). Eine Generalsanierung von WKA könnte die Lebensdauer auf weitere 15 Jahre verlängern (Wilburn 2011). Die wirtschaftliche Lebensdauer ist bei diesen Anlagen jedoch meist kürzer, weshalb sie meist früher abgebaut wer- den (z. B. aktuell durch Repowering bei WKA), um an anderen Orten (meist auch außerhalb Österreichs) im Sinne von Reuse wiederaufgebaut und -verwendet zu werden. Für diese Studie wird daher eine verkürzte Lebens- bzw. Nutzungsdauer von 20 Jahren für beide Systeme angenommen.

Aufgrund der geringen Abfallmengen kann auch ein hochwertiges Recycling noch nicht durchgeführt werden, da relevante Recyclingverfahren oftmals nur bei hoher Auslastung und großen ben werden können. In naher Zukunft werden die Abfallmengen unter Berücksichtigung der hohen Wachstumsraten von PVA und WKA jedoch drasKapazitätsgrößen wirtschaftlich betrie- tisch zunehmen und sich folglich auch die Verwertungswege ändern. Deshalb wird in dieser Studie zwischen dem „base case recycling“- und dem „highefficiency recycling"-Szenario unterschieden. Ersteres bezieht sich auf den Status quo der Verwertung und Entsorgung der Produktsysteme in Österreich. Das „high-efficiency recycling“-Szenario bezieht sich auf Recyclinglösungen, die aus heutiger Sicht schon technisch möglich wären, aber aus ökonomischen oder sonstigen Gründen derzeit nicht umgesetzt werden. Dies bezieht sich auch auf Möglichkeiten einer hochqualitativen Rückgewinnung von Sekundärressourcen.

Weiters ist anzumerken, dass sich in diesen Szenarien Recycling ausschließlich auf die stoffliche, nicht jedoch thermische Verwertung bezieht. In erster Linie betrifft dies Metalle, in zweiter Linie Kunststoffe aus vereinzelten Bauteilen, insofern sie manuell demontierbar und in größeren Mengen verfügbar sind (z.B. Rotorblätter). Die Recycling-Szenarien für PVA beziehen sich auf Angaben aus Ardente et al. (2019) und für WKA aus Vestas (2017) für den Status quo und aus Forschungsprojekten dokumentiert in Jensen and Skelton (2018) für das Recycling von Rotorblättern.

\section{Ergebnisse und Diskussion}

\subsection{Materialzusammensetzung}

Da der Marktmix an PVA in Österreich vorwiegend aus polykristallinen (74\%) und monokristallinen (26\%) Siliziumzellen besteht, wurden Dünnschichtmodule nicht berücksichtigt. Im Allgemeinen werden für diese Studie relevante siliziumbasierte PV-Zellen mit Bor dotiert, wobei mittels Phosphor-Diffusion Emitter an der Oberfläche der Wafer erzeugt werden. Eine Anti-Reflexionsschicht (blau bis blauschwarz gefärbtes Siliziumnitrit) auf der Vorderseite sowie Metallpasten, die Silber, Aluminium, Blei und Cadmium enthalten, stellen den elektrischen Kontakt der Vorder- und Rückseite der Zelle sicher. Eine Kunststofffolie aus Ethylvinylacetat (EVA) kapselt die Zellen hinter dem Glas ein, eine Tedlar ${ }^{\circledR}$-Folie wird als Rückseite des Moduls und ein Aluminiumrahmen zur Stabilisierung des Moduls verwendet (Kranert et al. 2012). Für die Rückseitenfolie wird eine Mischung aus Polyvinylfluorid (PVF) und Polyethylenterephthalat (PET) verwendet (De Wild-Scholten 2009, 2014). Die 
Glasabdeckung stellt hohe Anforderungen an Transparenz, Oberflächenreflexion und Festigkeit und besteht daher aus eisenarmem gehärtetem Glas. Zur Berechnung der Masse werden in dieser Arbeit eine Dicke von $4 \mathrm{~mm}$ und eine Dichte von $2,5 \mathrm{~g} / \mathrm{cm}^{3}$ angenommen. Sowohl das Glas als auch der Aluminiumrahmen müssen eine Lebensdauer von mehr als 20 Jahren unter schweren Umgebungsbedingungen im Freien garantieren. Kupfer wird für die Verbindung der Zellen untereinander und Zinn, Blei sowie Nickel werden für die Beschichtung verwendet. Eine Anschlussdose wird in der Regel auf der Rückseite von Modulen installiert und wird daher als Teil des PVModuls gezählt. Als massenrelevante Komponenten bei PV-Modulen fungieren Verbundglas als Hauptbestandteil, aber auch die Halterungen aus Aluminiumschienen. Ressourcenseitig sind Silber aus den Zellen sowie Silber und Gold aus den elektronischen Bauteilen von ökonomischer Bedeutung. Abb. 4 zeigt die charakteristische PVA-Materialzusammensetzung, die als Grundlage zur Massenberechnung des Bestands sowie der Abfallmengen diente.

Abb. 5 zeigt eine typische Materialienzusammensetzung einer WKA. Diese besteht aus einem Fundament und einem Turm, einer Gondel mit Generator, Transformatoren, einem Getriebe und Elektronik sowie einer Rotornabe mit drei Rotorblättern. Der Unterschied zwischen Turbinen mit Getriebe und getriebelosen Turbinen basiert auf der Technologie zur Umwandlung von Leistung zwischen Rotor und Turbine. Getriebelose Turbinen können mit Permanentmagneten aus Neodym ausgestattet werden (Luidold 2013). Neodym steht auf der EU-Liste für kritische Rohstoffe (European Commission 2020b), zählt zu den seltenen Erden und wird in Form von Neodym-Eisen-Bor (NdFeB) in getriebelosen Windenergieanlagen eingesetzt. Die spezifische Masse dieser Magneten liegt zwischen 0,5 und 1 Tonnen pro MW. Der Neodym-Gehalt beträgt im Magneten rund $30 \%$ und ergibt umgerechnet eine spezifische Masse von 0,15 bis 0,30 Tonnen Neodym pro MW (Luidold 2013). Hierbei ist anzumerken, dass der stark volatile Metallmarkt für $\mathrm{Nd} \mathrm{zu}$ einem relevanten Kostenfaktor für den Bau von Windkraftanlagen wurde. NdFeB-Magneten sind in Österreich allerdings derzeit nur in 9 von 1340 WKA eingebaut. Das Fundament und der Turm sind massenrelevante Komponenten bestehend aus Beton, Stahl oder einer Beton-StahlKonstruktion, deren Menge durch die
Nabenhöhe stark beeinflusst ist, die von 80 bis $173 \mathrm{~m}$ reichen kann, wobei der Rotordurchmesser in Österreich von 72 bis $124 \mathrm{~m}$ betragen kann (Biermayr et al. 2020; Winkelmeier et al. 2014). Wie in der PVA, spielen die eingesetzten elektronischen Komponenten auch in WKA hinsichtlich der eingebauten, metallischen Ressourcen eine große Rolle. In Abb. 5 wird die charakteristische Zusammensetzung einer WKA zusammenfassend dargestellt.

\subsection{Recycling}

Polykristalline siliziumbasierte PVA bestehen $\mathrm{zu}$ mehr als $80 \%$ aus Sicherheitsglas. Die Materialien (z. B. Siliziumzellen, vorder- und rückseitige Metallisationspaste) sind mit sehr hitzebeständigen Harzen verklebt, was eine Demontage erschwert und ein Recycling nur mit hohem Aufwand möglich macht. Der hohe Glasanteil in PVA ist für Verbundglasrecycler interessant. Allerdings ist das Recycling mit Verunreinigungen durch geklebte Zellen und schwermetallhaltige Pasten verbunden (Höller et al. 2016). Aufgrund der derzeit geringen Mengen an AltPVA werden diese Verunreinigungen nach dem „Verdünnungsprinzip“ während des Recyclings von Sicherheits-

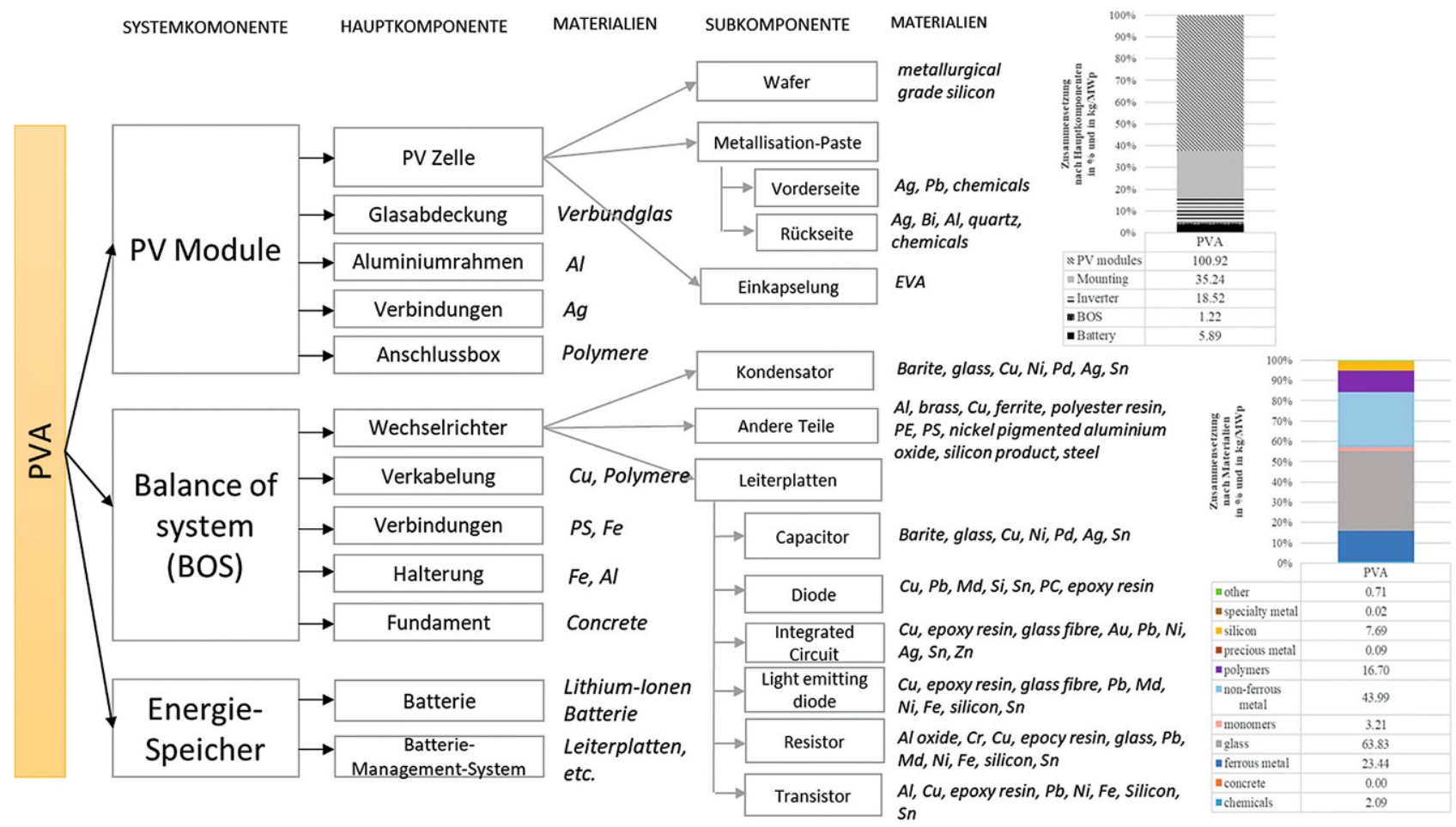

Abb. 4 Charakteristische Materialzusammensetzung einer PVA mit siliziumbasierten Solarzellen basierend auf Frischknecht et al. (2015), Louwen et al. (2015) und Hischier et al. (2007a; 2007b) 


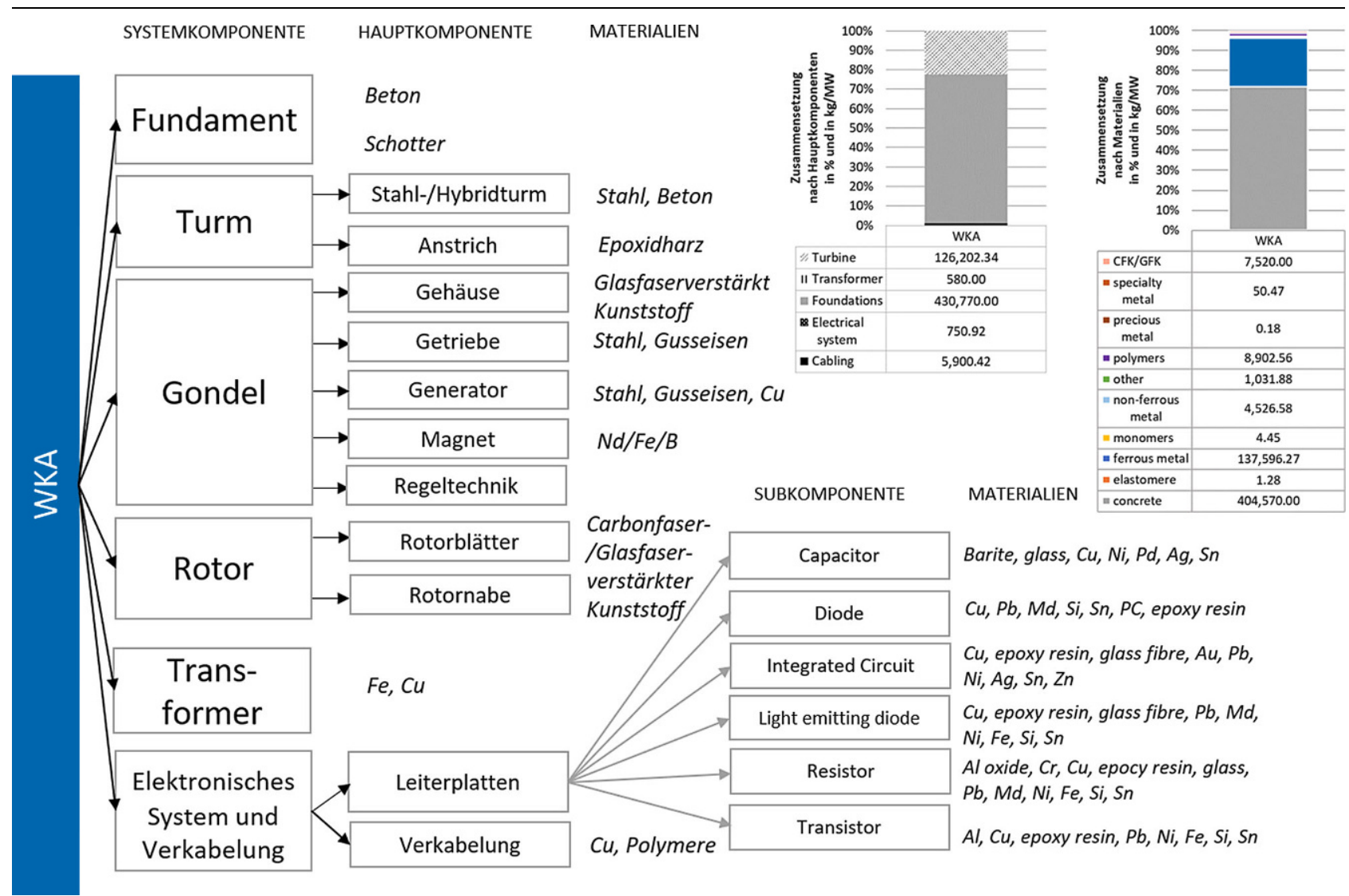

Abb. 5 Charakteristische Materialzusammensetzung einer WKA basierend auf Vestas (2017) sowie Hischier et al. (2007a; 2007b)

gläsern (vorwiegend Autoscheiben und Fenstergläser) derzeit noch toleriert. In Zukunft ist es bei steigenden Mengen jedoch fraglich, ob diese Verunreinigungen in der Verbundglasverwertung toleriert werden können. Die ordnungsgemäße Entsorgung von schwermetallhaltigen Bauteilen ist für viele Verwerter ein kritischer Aspekt, der die stoffliche Verwertung erschwert. Solche Gegebenheiten müssen in weiteren Studien näher analysiert werden, um in weiterer Folge die Recyclingquoten für PVA erhöhen zu können.

Eine häufig genutzte Verwertungsmöglichkeit der Sicherheitsgläser ist der Wiedereinsatz in der Glaswolleproduktion (Höller et al. 2016; Stolz et al. 2017). Die Recyclingtechnologien für siliziumbasierte PVA basieren auf physikalischem Abbruch und Trennung, z. B. bei der Firma Reiling und Exner Trenntechnik in Deutschland. Sie generieren Outputs, wie z.B. Schüttgutglasscherben, Aluminiumschrott und Kupferschrott (Stolz et al. 2017). Die Komponenten des „Balance of System“ (BOS, z.B. Montagestruktur und elektrische Installationen) werden in der Regel von den PV-Modulen getrennt und dem Me- tallrecycling (Halterungen, Schienen) oder EAG-Recycling (Anschlussdose, Wechselrichter etc.) zugeführt (Stolz et al. 2017). Halb-/Leitermaterialien aus Silizium oder Edelmetalle wie Silber werden aufgrund des relativ geringen Massenanteils derzeit nicht zurückgewonnen (Ardente et al. 2019). Eine Rückgewinnung solcher Wertmetalle könnte beispielsweise durch hydrometallurgische Verfahren erfolgen.

Zusammengefasst können PVA physikalisch, thermisch oder chemisch vorbehandelt werden, um den Glas-Solarzellen-Verbund aufzutrennen (Delaminierung). Des Weiteren werden mechanische und/oder chemische Methoden zur Nachbehandlung der gewonnenen Fraktionen angewandt, um am Ende mittels metallurgischer Verfahren wertvolle Industriemetalle rückgewinnen $\mathrm{zu}$ können (Fröhlich et al. 2017; Marwede et al. 2013).

Ein hocheffizientes Recycling von PV-Zellen wäre technisch möglich (z.B. zur Rückgewinnung von Glas in hoher Qualität, aber auch von Silizium), wird aber oft aus wirtschaftlichen Gründen unterlassen. Glasabfälle können rezykliert werden, um eine hohe Reinheit zu gewinnen, die dann für Anwendungen mittlerer bis hoher Qualität, wie z.B. die Herstellung von Flachglas, geeignet ist. Silizium kann durch Säurelaugung abgetrennt werden, um hochreines metallurgisches Silizium zu erhalten. Silber kann durch Elektrolyse auf Graphitstäben abgetrennt werden. Unter der Voraussetzung, dass die Anlagenkapazitäten ausgelastet werden können und ein Markt für Sekundärrohstoffe wie recyceltes Silizium erkennbar ist, wäre ein hocheffizientes Recycling realisierbar. Aluminium von Rahmen und internen Verbindern können durch automatisierte Demontage zerlegt und in hoher Reinheit weiterverarbeitet werden. Kupferabfälle von Kabeln und von Innenteilen des PV-Panels können ebenfalls rezykliert werden (Ardente et al. 2019).

Im Falle von WKA ist zu erwähnen, dass bislang erst ein Bruchteil der bestehenden Anlagen in Österreich abgebaut wurden - nach Einsiedler (2017) bis 2016 nur insgesamt 35 WKA. Ein verstärkter Rückbau wird allerdings in den nächsten Jahren aufgrund des Repowering erwartet. Die im Zuge des Repowerings abgebauten WKA können noch 
weiter genutzt werden und werden daher am Reuse-Markt für Windturbinen verkauft. So haben sich bereits einige Unternehmen auf den Verkauf von gebrauchten WKA spezialisiert (z.B. Green-Ener-Tech, Repowering Solutions, Enerpower, Windturbines). Die gebrauchten WKA sind günstiger und damit leistbarer für Schwellen- oder Entwicklungsländer. Vorteile sind dabei, dass jene Länder durch Technologietransfer profitieren und auch im Bereich der erneuerbaren Energiegewinnung Fuß fassen können. Durch die kürzere Beschaffungszeit und den geringeren Kapitaleinsatz kann das finanzielle Risiko reduziert werden, welches vor allem in wirtschaftlich und politisch unsicheren Ländern ausschlaggebend sein kann (Welstead et al. 2013). Es ist aber davon auszugehen, dass bei stärkerem Ausbau der WKA die Nachfrage nach alten WKA abnehmen wird. Vor allem größere (z.B. 3-MW-) Turbinen, sind für den Weitertransport und somit für Reuse weniger attraktiv als kleinere Turbinen. Neben einer Nutzung als Ersatzteilspender können sie dann nur noch für ein Recycling demontiert werden (Kaiser and Seitz 2014). Hierbei werden Verkabelungen, Flüssigkeiten, Schmierstoffe und Kühlmittel entfernt, die Drehdurchführung entkoppelt und anschließend die Rotorblätter und die Nabe mit einem Kran abgenommen. Gondel und Antriebsstrang werden ebenfalls demontiert. Stahltürme werden in ihre einzelnen Segmente zerlegt und können so auch leicht abtransportiert und wiederverwendet werden. Bei Hybridtürmen müssen die Betonsegmente von der Stahlkonstruktion aufgetrennt werden. Das Fundament wird je nach Vereinbarung im Boden belassen oder teilweise abgetragen (die obersten 1 bis $2 \mathrm{~m}$ ), um eine Wasserdurchlässigkeit des Bodens wiederherzustellen. Da das Fundament aus Stahlbeton und damit sehr beständig ist, kann es zumeist am Standort verbleiben, wobei Entsorgungskosten reduziert sowie geringe Umweltrisiken erwartet werden.

Da sich eine WKA aus sehr massereichen und recyclingfähigen Fraktionen aus Beton, Stahl sowie Kupfer und Aluminium zusammensetzt, kann eine sehr hohe Recyclingquote von 80 bis $90 \%$ erreicht werden (Seiler et al. 2014). Der Abbau ist wegen der Kupferverdrahtung im Generator wirtschaftlich attraktiv. Beton-Stahl-Konstruktionen können in größere Stücke gebrochen und zu Baurestmassen-Recyclinganlagen transportiert werden. Beton kann weiters mittels Prall- und Backenbrechern zerkleinert und beispielsweise als Betonsplitt in Frischbeton hinzugemischt werden. Kupferkabel werden mittels trockenmechanischer Zerkleinerung in Schneidmühlen und anschließender Dichtetrennung in Kupfer- und Kunststoffgranulate aufgetrennt. Das Recycling von Stahl, Kupfer, Aluminium und Eisen ermöglicht eine hochwertige Rückgewinnung von sekundären Rohstoffen, die in der Metallindustrie eingesetzt werden.

Anders verhält es sich mit den glasfaser- und carbonfaserverstärkten Kunststoffen (GFK bzw. CFK) bzw. Epoxidharzen, die in den Rotorblättern vermehrt Einsatz finden und für die es noch kaum kommerzielle Recyclingmethoden gibt. Diese Materialien unterliegen meist dem Downcycling und werden in Zementfabriken als aufbereitete Ersatzbrennstoffe zur Energiegewinnung eingesetzt. Es wird jedoch damit gerechnet, dass bei einem Anstieg der zu entsorgenden Rotorblätter andere Verwertungswege etabliert werden müssen, da die Mitverbrennung in Zementfabriken hinsichtlich eines unvollständigen Ausbrands oder Entstehung von schädlichen Verbrennungsprodukten (z.B. aromatische Kohlenwasserstoffe, Cyanwasserstoff, Kohlendioxid, Kohlenmonoxid etc.) auch mit Problemen einhergehen kann. Des Weiteren können durch den Verbrennungsvorgang halogenierte Flammschutzmittel, die GFK bzw. CFK zugegeben werden, unabsichtlich freigesetzt werden und so in den Abgasstrom übergehen. Somit können persistente Fasern den Verbrennungsprozess hindern, zudem auch die Menge an Flugasche vergrößern sowie Störungen in der Verbrennungsanlage auslösen (Beauson and Brøndsted 2016). Während des Recyclings müssen die Kompositmaterialien aus GFK und CFK in homogene Partikel aufgetrennt werden, wozu mechanische aber auch chemische Verfahren eingesetzt werden können. Beide Verfahren sind jedoch aufgrund der höheren Kosten des Sekundärmaterials im Vergleich zum Primärrohstoff derzeit nicht wirtschaftlich $\mathrm{zu}$ betreiben. Zudem sprechen der hohe Energieaufwand bei der Zerkleinerung sowie die großen Mengen an chemischen Abfällen noch gegen diese Verfahren. Es werden zurzeit daher andere Möglichkeiten zur Verwendung von ausgedienten Rotor- blättern bevorzugt. In Jensen and Skelton (2018) wurden Anwendungen im Brückenbau, für eine Konstruktion auf Kinderspielplätzen, für Möbel für den Innen- und Außenbereich sowie für langlebige Unterstände aufgezeigt. Die hauptsächlichen Hindernisse für diese Einsatzmöglichkeiten liegen derzeit an der Verfügbarkeit der Rotorblätter und den Einschränkungen im Design bzw. in der Weiterverarbeitung. In diesem Zusammenhang ist zu erwähnen, dass auch in Österreich bereits mittels Wettbewerb versucht wurde, innovative Ideen für ausrangierte Rotorblätter $\mathrm{zu}$ finden (z.B. Wettbewerb der Energie Burgenland zur Nachnutzung von alten Flügeln, Kurier, 10. Mai 2020).

In einem Forschungsprojekt publiziert in Jensen and Skelton (2018) wurden auch weitere mechanische sowie thermische Verwertungswege für Rotorblätter getestet (z.B. Spanplatten mit zerkleinerten GFK oder für Holzanstrich verwendeter Staub aus GFK). Abgesehen von energieintensiven Aufbereitungsschritten führen diese Anwendungen auch zu weiteren Problemfeldern beim Recycling. Jensen and Skelton (2018) folgern, dass eine Rückgewinnung der Materialien für den Einsatz in der Produktion derzeit noch nicht möglich ist. Es konnten zwar gute Fortschritte bei der Extraktion der Fasern mit guter Qualität festgestellt werden, jedoch nur im Labormaßstab mit sehr hohem Energieverbrauch und Kosten, die mit dem Preis der Neuware derzeit nicht konkurrieren können. Für das gegenständliche Szenario des „high efficiency“-Recyclings wird daher nur eine Recyclingrate von $50 \%$ angenommen.

Sowohl in einer PVA als auch in einer WKA sind elektronische Bauteile, wie Kondensatoren, Leiterplatten, Dioden, Transformatoren etc., verbaut, die über die EAG-Verwertung rezykliert werden können. Diese Elektroaltgeräte enthalten neben Aluminium, Stahl und Kupfer auch Edel- und Sondermetalle wie Tantal. Der Recyclingweg ist jedoch eher die Kupfermetallurgie, die eine hohe Recyclingausbeute für Kupfer, aber nicht für Tantal garantiert (Luidold 2013). Die Recyclingquote von kritischen Metallen wie Tantal oder Platingruppenmetalle ist derzeit noch niedrig und erfolgt derzeit eher im Falle von sortenreinen Produktionsabfällen oder anderen speziellen Segmenten wie Karbiden. Für eine kreislauforientierte Wirtschaft und zur Versorgungssicher- 
heit wird es jedoch notwendig sein, die Recyclingeffizienz zu erhöhen. Deshalb wird im "high efficiency Recycling“Szenario auch mit einer Rückgewinnung von Gold, Silber und Zinn gerechnet. Das Recyclingpotenzial von Sondermetallen wie Indium, Gallium, Germanium und Tantal wurde nicht angeführt, da die derzeitigen Recyclingraten sehr niedrig und hauptsächlich auf Produktionsabfälle beschränkt sind. Der Metallgehalt in den Produkten ist relativ niedrig. So liegt beispielsweise der Tantal-Gehalt von Kondensatoren, die im Wechselrichter eingebaut sind, bei ca. 0,005 g pro installiertem Wp. Darüber hinaus mangelt es derzeit noch an effizienten Recyclingtechnologien, um Sondermetalle wie seltene Erdmetalle rückgewinnen zu können (UNEP 2011). Eine Abschätzung der zukünftigen Recyclingmöglichkeiten für diese Sondermetalle konnte aus diesen Gründen nicht getroffen werden. Eine $\mathrm{Zu}$ sammenfassung der berücksichtigten Metalle und sonstigen recyclingfähigen Materialien für die einzelnen Szenarien wird in Tab. 1 dargestellt.

Tab. 1 Zusammenfassung der berücksichtigten recyclingfähigen Materialien für die einzelnen Szenarien unter Angabe der Recycling-Rate (RR)

\begin{tabular}{|c|c|c|}
\hline & Base case Recycling & High-efficiency Recycling \\
\hline Definition & $\begin{array}{l}\text { Status quo, Fokus auf massenba- } \\
\text { sierte Rückgewinnungsrate }\end{array}$ & $\begin{array}{l}\text { Aus heutiger Sicht technisch möglich, } \\
\text { Fokus auf material bezogene Rückgewinnungsrate }\end{array}$ \\
\hline \multicolumn{3}{|l|}{ PVA } \\
\hline Aluminium & $\begin{array}{l}\text { RR 92\%: Al-Rahmen wird ma- } \\
\text { nuell entfernt, äquivalent zu } \\
\text { Primär-Aluminium }\end{array}$ & $\begin{array}{l}\text { RR 94\%: Al-Rahmen und Al von weiteren Verbindun- } \\
\text { gen (automatische Trennung und weitere Verarbei- } \\
\text { tung), äquivalent zu Primär-Aluminium }{ }^{\mathrm{a}}\end{array}$ \\
\hline Kupfer & $\begin{array}{l}\text { RR } 72 \% \text { : Kupferschrott aus Ka- } \\
\text { beln äquivalent zu Primär-Kupfer }\end{array}$ & RR $90 \%$ : aus Kabeln und sonstigen Verbindungen ${ }^{\mathrm{a}}$ \\
\hline Glas & $\begin{array}{l}\text { RR 9\%: Glasschrott/wolle; äqui- } \\
\text { valent zu Anwendungen mit } \\
\text { niedriger Qualität }^{\mathrm{a}}\end{array}$ & $\begin{array}{l}\text { RR } 88 \% \text { : Glas wird durch ein hochselektives Verfah- } \\
\text { ren abgetrennt, um eine hohe Reinheit zu erhalten. } \\
\text { Antimon im Glas geht verloren }{ }^{\mathrm{a}}\end{array}$ \\
\hline Silizium & $\operatorname{RR} 0 \%^{a}$ & $\begin{array}{l}\text { RR } 95 \% \text { : Durch Säureauslaugung abgetrenntes Sili- } \\
\text { zium, um hochreines Silizium-Metall von metallurgi- } \\
\text { scher Qualität zu erhalten }^{a}\end{array}$ \\
\hline Silber & $\mathrm{RR} 0 \%^{\mathrm{a}}$ & $\begin{array}{l}\text { RR } 94 \% \text { : Silber durch Elektrolyse auf Graphitstangen } \\
\text { getrennt, äquivalent zu Primärmaterial }\end{array}$ \\
\hline EAG & Nur Al, $\mathrm{Cu}, \mathrm{Fe}$ aus EAG ${ }^{\mathrm{b}}$ & $+\mathrm{Au}, \mathrm{Ag}, \mathrm{Sn}{ }^{\mathrm{b}}$ \\
\hline \multicolumn{3}{|l|}{ WKA } \\
\hline Beton & $\begin{array}{l}\text { RR } 92 \% \text { Beton aus dem } \\
\text { Fundament }{ }^{\mathrm{C}}\end{array}$ & RR $92 \%$ Beton aus dem Fundament ${ }^{c}$ \\
\hline Stahl & RR $92 \%^{\mathrm{C}}$ & RR $92 \%$ c \\
\hline Aluminium & RR $92 \%^{\mathrm{c}}$ & RR $92 \%{ }^{c}$ \\
\hline Kupfer & RR $95 \%{ }^{c}$ & RR $95 \%{ }^{\mathrm{C}}$ \\
\hline GFK/CFK & $\mathrm{RR} 0 \%^{\mathrm{b}}$ & RR $50 \%{ }^{b}$ \\
\hline EAG & Nur $\mathrm{Al}, \mathrm{Cu}, \mathrm{Fe}$ aus $\mathrm{EAG}^{\mathrm{b}}$ & $+\mathrm{Au}, \mathrm{Ag}, \mathrm{Sn}^{\mathrm{b}}$ \\
\hline
\end{tabular}

nen des Weiteren auch mögliche Wege zur stofflichen Verwertung etabliert werden (z.B. Kunststoff-Gehäuse, die leicht demontierbar sind; chemisches Recycling der Rotorblätter etc.).

In Abb. 7 ist zu erkennen, dass der Großteil an recyclingfähigen Stoffen im Bereich des Betons zu erwarten ist, welche zu Frischbeton zugemischt oder im Straßenbau verwendet werden können. Ein Großteil an Sekundärressourcen ist auch im Bereich der Eisenmetalle zu erwarten, dies vor allem niedriglegierter Stahl, aber auch Gusseisen. Da die Ausbaurate im Transition-Szenario um ein Vielfaches höher ist als im WEM-Szenario, gibt es hier mengenmäßig große Unterschiede. Im WEM-Szenario werden für 2050 Eisenmetalle um 7,52 Tonnen erwartet, im Transition-Szenario um 43,7 Tonnen. Da bezüglich der Recyclingrate von Beton und Eisenmetalle kein Unterschied zwischen „base case reycling“ (SR_1) und „high-efficient recycling“ (SR_2) gemacht wurde, gibt es hier keine Differenzen. Anders sieht es bei den Edelmetallen aus, wo im Szenario „high-efficient recycling“ zwischen 9 und $55 \mathrm{~g}$ zu erwarten sind 92 Massen-\% sind davon Silber. Das Recycling von Magneten (hier in der Gruppe der Sondermetalle) mit jährlich $3 \mathrm{~kg}$ bzw. $17,4 \mathrm{~kg}$ je nach Ausbaurate ist ungewiss. Magnete, bestehend aus seltenen Erden wie Neodymium und Dysprosium, werden in Vorrichtungen im Turm benötigt. Die Rolle von CFK bzw. GFK zeigt sich bei Annahme einer Recyclingrate von $50 \%$ mit einer Menge von 223 bis $1300 \mathrm{~kg}$ für das Jahr 2050 .

\section{Schlussfolgerung und Ausblick}

Der Ausbau von erneuerbarer Energie hat einen erheblichen Anstieg unseres Ressourcenverbrauchs zur Folge. Im Sinne des EU-Kreislaufwirtschaftspakets sollen Materialien und v.a. kritische Rohstoffe rückgeführt werden, um so Ressourcen zu schonen und von Primärrohstoffimporten unabhängiger $\mathrm{zu}$ werden. Hierbei sind recyclingfähige Materialien eine wichtige Quelle für Sekundärressourcen. Ein hochwertiges Recycling erhöht die Bandbreite der Einsatzmöglichkeiten für Sekundärrohstoffe und leistet einen erheblichen Beitrag zur Versorgungssicherheit. In diesem Beitrag wird unter Zuhilfenahme einer Materialflussanalyse (MFA) gezeigt, wie viele und welche Rohstoffe in den jetzigen und zukünftigen PVA und WKA in Österreich verbaut sind 
PVA

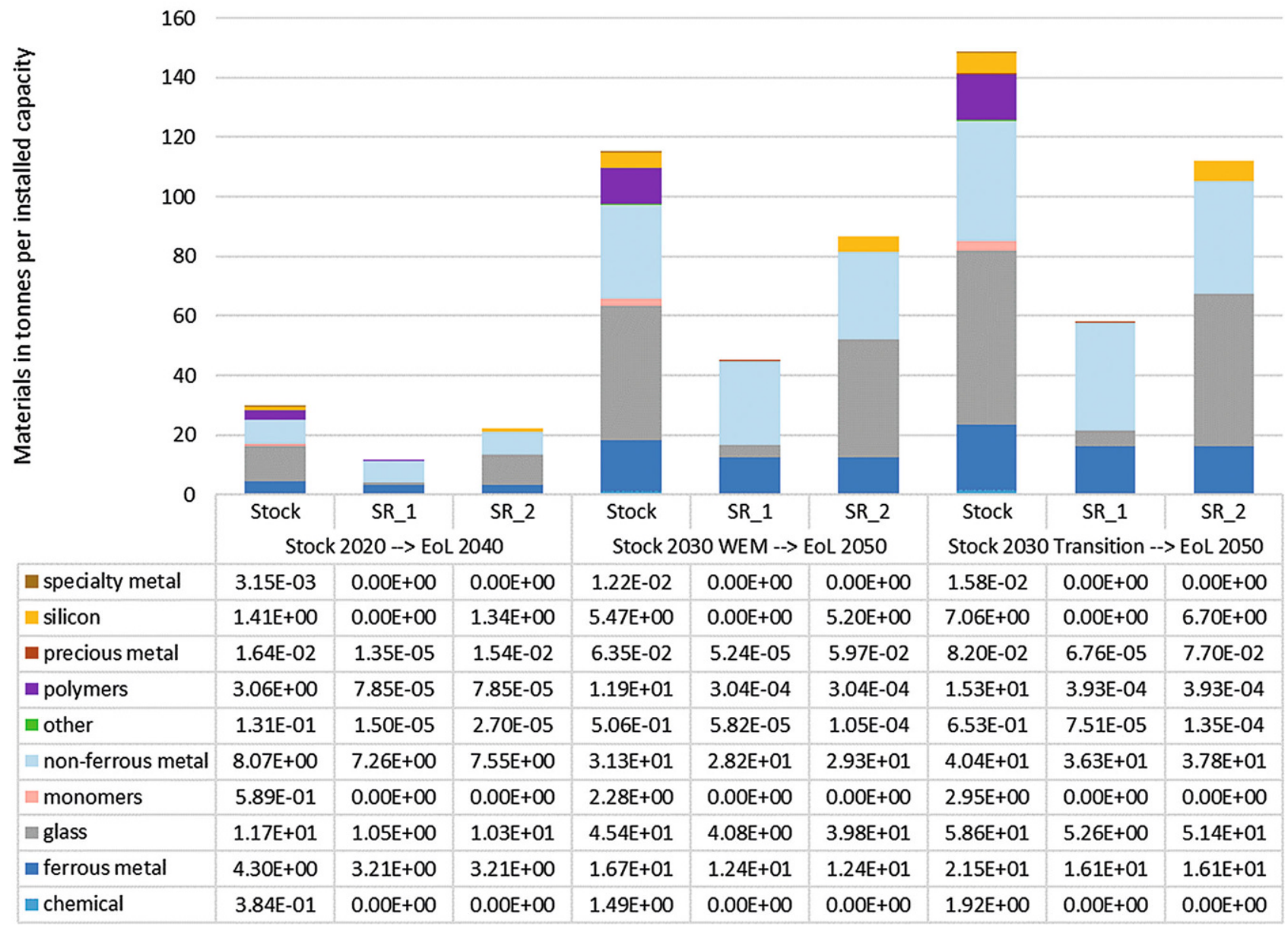

Abb. 6 Ermitteltes Sekundärressourcenpotenzial in Tonnen der jährlich installierten Menge an PVA in Österreich

und bei Ende der Nutzungsdauer für eine Rohstoff-Rückgewinnung potenziell zur Verfügung stehen könnten. Die MFA zeigt, dass ein großes Potenzial zur Rückgewinnung von Sekundärrohstoffen, besonders für massenrelevante Teile der Anlagen wie Betonfundament, Aluminiumhalterungen, Stahlträger und Verbundglas, besteht. Sie zeigt aber auch quantitative Abschätzungen für weniger massenrelevante, jedoch hochwertige Materialien, die bei einer hochwertigen Rückgewinnung großes Potenzial zur Erreichung einer Versorgungssicherheit in Europa haben.

Im Hinblick auf weiterführende Studien wird darauf verwiesen, dass die Daten für eine solche MFA in einer bestimmten Detailtiefe verfügbar sein müssen, um feststellen zu können, in welchen Haupt- und Subkomponenten welche Materialien bzw. Stoffe eingesetzt werden. Dabei ist es oftmals nicht ausreichend, ausschließlich auf Daten aus publizierten Ökobilanz-Stu- dien zurückzugreifen, da diese meist nur Daten auf Ebene der Hauptkomponenten oder der Stoffgruppen dokumentieren. Deshalb wurden in diesem Beitrag auch Daten aus Ökobilanz-Datenbanken (Ecoinvent Centre 2019) zu bestimmten Bauteilen (z.B. Leiterplatten, Dioden etc.) herangezogen, um die Produktsysteme zu komplementieren. Diese Ergänzungen waren erforderlich, um insbesondere verbaute Eisen- und Nichteisenmetalle sowie Edel- und Sondermetalle in elektronischen Bauteilen zu identifizieren und quantifizieren.

Diese Studie zeigt auch auf, dass die Rückgewinnung von Sekundärressourcen aus WKA und PVA vielen Herausforderungen unterliegt. Zum einen sind die Rohstoffe in langlebigen Wirtschaftsgütern gebunden. Die Lebensdauer von PVA und WKA liegt bei mindestens 20 Jahren und oftmals mehr. Des Weiteren ist ihr Wiederverwendungspotenzial (Abbau und Aufbau sowie Nutzung an einem anderen
Standort) sehr hoch, obwohl es mit Verlusten in der spezifischen Leistung einhergeht, die jedoch aufgrund der niedrigen Anschaffungskosten in der Regel toleriert werden. Dies ergibt eine erhebliche zeitliche Verzögerung zwischen Herstellung und Demontage, wobei die Anlagenteile teilweise exportiert werden und mehrmals den Besitzer wechseln. Zum anderen sind die Sortier- und Recyclingtechnologien für viele Rohstoffe noch nicht zu wettbewerbsfähigen Kosten verfügbar (insbesondere bei GFK oder CFK aus Rotorblättern oder hochwertigem Glas aus Verbundglas von PV-Modulen).

Zusammengefasst fallen derzeit noch geringe Mengen an Abfällen aus diesen Sektoren in Österreich an, daher sind Sammel- sowie Recyclingsysteme für solche Anlagen noch nicht vollständig etabliert und es fehlen außerdem Erfahrungswerte. Nichtsdestotrotz werden in naher Zukunft - unter Berücksichtigung der hohen Wachstumsra- 


\section{WKA}

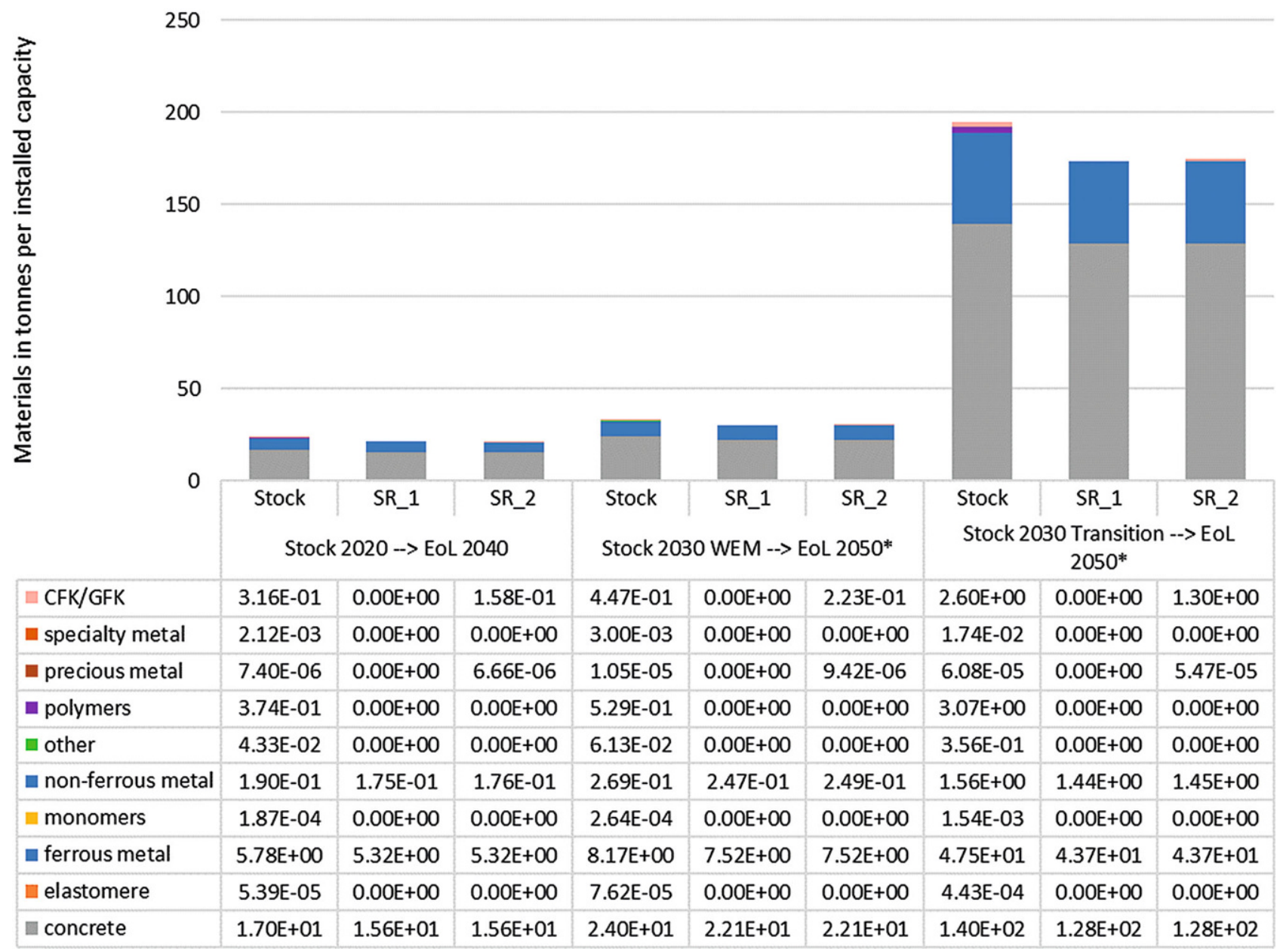

Abb. 7 Ermitteltes Sekundärressourcenpotenzial in Tonnen der jährlich installierten Menge an WKA in Österreich

ten von PVA und WKA - sowohl der Ressourcenverbrauch als auch das Abfallaufkommen und in weiterer Folge auch das Sekundärressourcenpotenzial stetig wachsen. Markbedingt wird dies bei PVA frühestens 2030 der Fall sein. Die Mengenentwicklung von AltWKA ist derzeit mit hohen Unsicherheiten behaftet, da derzeit die Praktiken Repowering bzw. Reuse (Weiterverkauf) bevorzugt angewandt werden, bevor Alt-WKA stofflich verwertet werden können. Hinsichtlich effizienterer Ressourcennutzung und der höheren Stromproduktion durch erneuerbare Energie ist die Wiederverwendung von WKA und PKA grundsätzlich positiv $\mathrm{zu}$ betrachten. Mit Hinblick auf eine Nachhaltigkeitsbewertung müssen die Herausforderungen im Recycling und in der Entsorgung unbedingt mitberücksichtigt werden, welche sich auf einen späteren Zeitpunkt und meist in ein anderes Land verlagern. Es wird daher mehr denn je von Bedeutung sein, „Design for Reuse“ aber auch „Design for Recycling“ für diese Produktgruppen voranzutreiben, um vor allem eine manuelle Demontage sowie stoffliche Verwertung $\mathrm{zu}$ erleichtern. Weiterer Forschungsbedarf besteht in der Beurteilung der Nachhaltigkeit verschiedener Verwertungswege (stofflich und thermisch) unter Bezugnahme auf Versorgungssicherheit aber auch Effizienz. So kann ein Verwertungsweg, der wirtschaftlich nicht umsetzbar aber nachhaltiger ist als andere Wege, durch Förderinstrumente (z.B. Begünstigungen, Fonds) gezielt unterstützt werden. Eine Betrachtung des gesamten Lebenszyklus sowie aller In- und Outputs diverser Prozesse (insbesondere Energieaufwand, chemische Abfälle) ist in zukünftigen Studien zu berücksichtigen. Schlussendlich bedarf es oftmals spezieller Recyclingverfahrenskombinationen mit hohem Durchsatz, um diese effizient und v.a. wirtschaftlich betreiben zu können. Der Markt in Österreich in Bezug auf PVA und WKA reicht trotz der stark ansteigenden Ausbaurate möglicherweise nicht aus, um bestimmte Recyclinganlagen zu betreiben. Bei diesen langlebigen und komplexen Produkten wird es daher mehr denn je wichtig sein, Wertschöpfungsketten europaweit zu vernetzen, damit WKA und PKA nicht nur in Bezug auf die Energieerzeugung, sondern auch in Bezug auf die Materialnutzung und deren Recyclingfähigkeit als umweltschonend gelten können.

Danksagung Dieser Beitrag wurde im Rahmen des Projektes ROSE-Trans („The Role of Secondary Resources in the Austrian Energy Transition“, Projekt-Nr. 18054) gefördert vom Jubilä- 
umsfonds der Österreichischen Nationalbank erstellt. Das Projekt läuft von April 2019 bis Februar 2021 unter der Leitung des Österreichischen Instituts für Wirtschaftsforschung. Die Autoren bedanken sich bei Mark Sommer und Ina Meyer für die Bereitstellung der Daten aus den Energieszenarien.

Funding Open access funding provided by University of Natural Resources and Life Sciences Vienna (BOKU).

Open Access Dieser Artikel wird unter der Creative Commons Namensnen- nung 4.0 International Lizenz veröffentlicht, welche die Nutzung, Vervielfältigung, Bearbeitung, Verbreitung und Wiedergabe in jeglichem Medium und Format erlaubt, sofern Sie den/die ursprünglichen Autor(en) und die Quelle ordnungsgemäß nennen, einen Link zur Creative Commons Lizenz beifügen und angeben, ob Änderungen vorgenommen wurden.

Die in diesem Artikel enthaltenen Bilder und sonstiges Drittmaterial unterliegen ebenfalls der genannten Creative Commons Lizenz, sofern sich aus der Abbildungslegende nichts anderes ergibt. Sofern das betreffende Material nicht unter der genannten Creative Commons Lizenz steht und die betreffende Handlung nicht nach gesetzlichen Vorschriften erlaubt ist, ist für die oben aufgeführten Weiterverwendungen des Materials die Einwilligung des jeweiligen Rechteinhabers einzuholen.

Weitere Details zur Lizenz entnehmen Sie bitte der Lizenzinformation auf http://creativecommons.org/licenses/ by/4.0/deed.de.

\section{Literatur}

Ardente F, Beccali M., Cellura M., Lo Brano V. (2008): Energy performances and life cycle assessment of an Italian wind farm Renewable and Sustainable Energy Reviews 12:200-217 https:// doi.org/10.1016/j.rser.2006.05.013

Ardente F., Latunussa C.E.L., Blengini G.A. (2019): Resource efficient recovery of critica and precious metals from waste silicon PV panel recycling Waste Management 91:156-167 https://doi.org/10.1016/j.wasman.2019.04.059 Beauson J., Brøndsted P. (2016): Wind Turbine Blades: An End of Life Perspective. In: MAREWINT, 421-432.

Biermayr P. et al. (2020): Innovative Energietechnologien in Österreich Marktentwicklung 2019, Berichte aus Energie- und Umweltforschung, vol 14/2020. Bundesministerium für Klimaschutz, Umwelt, Energie, Mobilität, Innovation und Technologie (BMK), Wien

Bio Intelligence Service (2011): Study on Photovoltaic Panels Supplementing the Impact Assessment for a Recast of the WEEE Directive.

Ecoinvent Centre (2019): Ecoinvent database v3.6.

Einsiedler D. (2017): End of Life Wege von Windkraftanlagen in Österreich: Herausforderungen und Chancen beim Recycling, University of $\mathrm{Na}$ tural Resources and Life Sciences, Institute of Safety and Risk Assessment, Wien

Europäische Kommission (2015): Den Kreislau schließen - Ein Aktionsplan der EU für die Kreislaufwirtschaft. Brüssel

European Commission (2020a): Circular Economy Action Plan. For a cleaner and more competitive Europe. \#EUGreenDeal.

European Commission (2020b): Study on the EU's list of Critical Raw Materials - Final Report. Fischer L., Leohnhartsberger K. (2019): Analyse der Marktentwicklung von PV-Heimspeichersystemen in Österreich. FH Technikum Wien, F\&E Schwerpunkt Renewable Energy Systems,

Frischknecht R. et al. (2015): Life Cycle Inventories and Life Cycle Assessments of Photovoltaic Systems. Report IEA-PVPS T12-04:2015

Fröhlich P., Lorenz T., Martin G., Brett B., Bertau M. (2017): Valuable Metals-Recovery Processes, Current Trends, and Recycling Strategies Angewandte Chemie International Edition 56:2544-2580 doi:doi:10.1002/anie.201605417 Garrett P., Rønde K. (2014): Life Cycle Assessment of Electricity Production from an onshore V126-3.3 MW Wind Plant. Aarhus, Denmark

Guezuraga B., Zauner R., Pölz W. (2012): Life cycle assessment of two different 2 MW class wind turbines Renewable Energy 37:37-44 https:// doi. org/10.1016/j.renene.2011.05.008
Hischier R, Classen M., Lehmann M., Scharnhorst W. (2007a): Life cycle inventories of Electric and Electronic Equipment: Production, Use and Disposal. Part I components. ecoinvent report No. 18. Empa / Technology \& Society Lab, Swiss Centre for Life Cycle Inventories ${ }_{\text {n }}$ Dübendorf, Switzerland

Hischier R., Classen M., Lehmann M., Scharnhorst W. (2007b): Life cycle inventories of Electric and Electronic Equipment: Production, Use and Disposal. Part II modules. ecoinvent report No. 18. . Empa / Technology \& Society Lab, Swiss Centre for Life Cycle Inventories, Dübendorf, Switzerland

Höller R, Wimmer A, Scherhaufer S., Part F, Salhofer S. (2016): Sammel- und Verwertungsstrategie für Photovoltaik-Module in Oberösterreich, Im Auftrag der OÖ Landes-Abfallverwertungsunternehmen AG, FH OÖ Forschung und Entwicklungs $\mathrm{GmbH}$, Universität für Bodenkultur Wien, Wels/Wien

Jensen J.P., Skelton K. (2018): Wind turbine blade recycling: Experiences, challenges and possibilities in a circular economy Renewable and Sustainable Energy Reviews 97:165-176 https:// doi.org/10.1016/j.rser.2018.08.041

Jungbluth, N., Stucki, M., Frischknecht, R. 2009): Photovoltaics, in: Dones, Ree (Ed) Sachbilanzen von Energiesystemen: Grundlagen für den ökologischen Vergleich von Energiesystemen und den Einbezug von Energiesystemen in Ökobilanzen für die Schweiz. ecoinvent report No. 6-XII, Swiss Centre for Life Cycle Inventories. Dübendorf, $\mathrm{CH}$

Kaiser O.S., Seitz H. (2014): Ressourceneffizienz von Windenergieanlagen. VDI ZRE Publikationen: Kurzanalyse Nr. 9. VDI Technologiezentrum $\mathrm{GmbH}$, Berlin

Kirchner M., Sommer M., Kratena K. (2017) Distributional impacts of a CO2 fuel tax on different household income quintiles in Austria. Paper presented at the 2017 International Energy Workshop July 12-14, 2017, College park, Maryland,

Kranert M., Metzger J., Koch M., Fisch K. (2012) Photovoltaikmodule - Umweltfreundlichkeit und Recyclingmöglichkeiten. Abschlussbericht. Kratena K, Sommer M. (2014): Policy implications of resource constraints on the European economy WWWforEurope Policy Brief no 6, November

Louwen A, van Sark W.G.J.H.M., Schropp R.E.I. Turkenburg W.C., Faaij A.P.C. (2015): Life-cycle greenhouse gas emissions and energy payback time of current and prospective silicon heterojunction solar cell designs Progress in Photovol- taics: Research and Applications 23:1406-1428 https://doi.org/10.1002/pip.2540

Luidold S. (2013): Kritische Rohstoffe für die Hochtechnologieanwendung in Osterreich. Im Auftrag des Bundesministeriums für Verkehr, Innovation und Technologie, Wien

Marwede M., Berger W., Schlummer M., Mäurer A., Reller A. (2013): Recycling paths for thinfilm chalcogenide photovoltaic waste-Current feasible processes Renewable Energy 55:220-229 https://doi.org/10.1016/j.renene.2012.12.038 Meyer I., Sommer M., Kratena K. (2018): Energy Scenarios 2050 for Austria. Austrian Institute of Economic Research-WIFO, Centre of Economic Scenario Analysis and Research-CESAR, Vienna Meyer I., Sommer M., Kratena K. (2020): How to reach Paris: a comprehensive long-term energyeconomy scenario for Austria. In: Zachariadis T, mic Instruments for a Low-carbon Future Critical Issues in Environmental Taxation. Edward Elgar Publishing, Cheltenham, UK, pp S.127-140. https://doi.org/10.4337/9781839109911.00023

Olson C., De Wild-Scholten M., Scherff M., Ribeyron P.-J. (2011): Life cycle assessment of heterojunction solar cells and modules. In: Ossenbrink H., Jäger-Waldau A., Helm P. (eds): 26th European Photovoltaic Solar Energy Conference and Exhibition, EU PVSEC, Hamburg

Österreichische Bundesregierung (2020): Aus Verantwortung für Österreich. Regierungsprogramm 2020-2024. Die neue Volkspartei, Die Grünen - Die Grüne Alternative,

Photovoltaic Austria (2020): Die österreichische Photovoltaik \& Speicher-Branche in Zahlen Seiler E., Bilitewski B., Woidasky J. (2014): Recycling von Windkraftanlagen. Konferenzbeitrag. Fraunhofer-Institut für Chemische Technologie ICT Pfinztal und Technische Universität Dresden.

Stolz P., Frischknecht R., Wambach K., Sinha P. Heath G. (2017): Life Cycle Assessment of Current Photovoltaic Module Recycling. Report IEA PVPS T12-13:2018.

Umweltbundesamt (2018): Klimaschutzbericht 2018.

Umweltbundesamt (2019): Klimaschutzbericht 2019.

UNEP (2011): Recycling rates of metals.

Vestas (2015): Life Cycle Assessment of Electricity Production from an onshore V110-2.0 MW Wind Plant. Aaerhus, Denmark

Vestas (2017): Life Cycle Assessment of Electricity Production from an onshore V112-3.45 MW Wind Plant.

Welstead J., Hirst R., Keogh D., G. R, Bainsfair R. (2013): Research and guidance on restoration 


\section{Originalarbeit}

and decommissioning of onshore wind farms. Scottish Natural Heritage Commissioned Report No. 591.

Wilburn D.R. (2011): Wind energy in the United States and materials required for the land-based wind turbine industry from 2010 through 2030 . U.S. Geological Survey, Reston, VA. https://doi. org/10.3133/sir20115036
De Wild-Scholten M. (2009): Renewable and Sustainable, Presentation at the CrystalClear final event. SmartGreenScans, Munich, DE De Wild-Scholten M. (2014): Life Cycle Assessment of Photovoltaics Status 2011, Part 1 Data Collection, report published by SmartGreenScans.

Winkelmeier H., Krenn A., Zimmer F. (2014): Das realisierbare Windpotential Österreichs für 2020 und 2030
Hinweis des Verlags Der Verlag bleibt in Hinblick auf geografische Zuordnungen und Gebietsbezeichnungen in veröffentlichten Karten und Institutsadressen neutral. 